\title{
The commercial pig as a model of spontaneously-occurring osteoarthritis
}

\author{
Mhairi A. Macfadyen' ${ }^{1}$, Zoe Daniel', Sara Kelly ${ }^{1}$, Tim Parr ${ }^{1}$, John M. Brameld ${ }^{1}$, Andrew J. Murton ${ }^{1,2,3 \dagger}$ and \\ Simon W. Jones ${ }^{4^{*}+}$ (D)
}

\begin{abstract}
Background: Preclinical osteoarthritis models where damage occurs spontaneously may better reflect the initiation and development of human osteoarthritis. The aim was to assess the commercial pig as a model of spontaneous osteoarthritis development by examining pain-associated behaviour, joint cartilage integrity, as well as the use of porcine cartilage explants and isolated chondrocytes and osteoblasts for ex vivo and in vitro studies.

Methods: Female pigs (Large white $x$ Landrace $\times$ Duroc) were examined at different ages from 6 weeks to 3-4 years old. Lameness was assessed as a marker of pain-associated behaviour. Femorotibial joint cartilage integrity was determined by chondropathy scoring and histological staining of proteoglycan. IL-6 production and proteoglycan degradation was assessed in cartilage explants and primary porcine chondrocytes by ELISA and DMMB assay. Primary porcine osteoblasts from damaged and non-damaged joints, as determined by chondropathy scoring, were assessed for mineralisation, proliferative and mitochondrial function as a marker of metabolic capacity.

Results: Pigs aged 80 weeks and older exhibited lameness. Osteoarthritic lesions in femoral condyle and tibial plateau cartilage were apparent from 40 weeks and increased in severity with age up to 3-4 years old. Cartilage from damaged joints exhibited proteoglycan loss, which positively correlated with chondropathy score. Stimulation of porcine cartilage explants and primary chondrocytes with either IL-1 $\beta$ or visfatin induced IL-6 production and proteoglycan degradation. Primary porcine osteoblasts from damaged joints exhibited reduced proliferative, mineralisation, and metabolic capacity.
\end{abstract}

Conclusion: In conclusion, the commercial pig represents an alternative model of spontaneous osteoarthritis and an excellent source of tissue for in vitro and ex vivo studies.

Keywords: Osteoarthritis, Osteoblast, Chondropathy, Pig, Chondrocyte

\section{Background}

Osteoarthritis $(\mathrm{OA})$ is an age-related joint disorder and the most common degenerative joint disorder in the World [1]. Characterised by degenerative loss of the articular cartilage, joint space narrowing, synovial inflammation and bone remodelling [2], it is a leading cause of disability and pain. Unfortunately, at present there are no available disease modifying OA drugs (DMOADs) [2, 3]. As an ageing population OA is a major health concern since it limits independence, reduces an individual's quality of life and puts

\footnotetext{
* Correspondence: s.w.jones@bham.ac.uk

${ }^{+}$Andrew J. Murton and Simon W. Jones contributed equally to this work.

${ }^{4}$ Institute of Inflammation and Ageing, MRC-ARUK Centre for Musculoskeletal

Ageing Research, School of Immunity, University of Birmingham,

Birmingham, UK

Full list of author information is available at the end of the article
}

additional pressure on healthcare systems and elderly support services $[4,5]$.

Critically, the development of DMOADs has been hampered by a lack of understanding of the joint pathology in early OA. Unfortunately, investigating early OA joint pathology in humans is inherently difficult. Synovial tissue and synovial joint fluid can be collected from early OA patients by arthroscopy procedures. However, OA diseased cartilage and bone tissue of sufficient quantity can generally only be obtained from end-stage diseased patients who are undergoing elective joint replacement surgery. Cartilage tissue from end-stage OA patients is often highly degraded and is therefore of questionable relevance to our understanding of the central pathways that underpin the initiation and development of early degenerative changes

(c) The Author(s). 2019 Open Access This article is distributed under the terms of the Creative Commons Attribution 4.0 International License (http://creativecommons.org/licenses/by/4.0/), which permits unrestricted use, distribution, and reproduction in any medium, provided you give appropriate credit to the original author(s) and the source, provide a link to the Creative Commons license, and indicate if changes were made. The Creative Commons Public Domain Dedication waiver (http://creativecommons.org/publicdomain/zero/1.0/) applies to the data made available in this article, unless otherwise stated. 
in the human OA joint. Furthermore, many of the in vivo preclinical models that are utilised are artificial models [6] where OA is experimentally induced either chemically or surgically. Surgical induction of OA is achieved by destabilising the joint through the surgical damage of joint ligaments, for example anterior cruciate ligament tear in the dog [7] or more recently in mice through the destabilisation of the medial meniscus (DMM model) [8, 9]. Although these models are effective in inducing joint damage, they more likely reflect changes seen in traumatically induced OA, where an injury has triggered further damage, rather than age-related $\mathrm{OA}[6,8,10]$.

For studying age-related human OA onset, animal models that spontaneously develop OA are likely to be more translatable. Such translation is critical to the development of new OA drugs. Indeed, the greatest reason for late-stage failure of candidate drugs can be traced back to failure of preclinical target validation studies to translate in the clinic [11]. In this regard, the Dunkin Hartley guinea pig is a notable example [12]. These animals develop OA without surgical, chemical or environmental manipulation $[12,13]$. However, the quantity of joint tissue for ex vivo and in vitro studies can be limiting. Spontaneous development of $\mathrm{OA}$ has also been studied in large animals including horse [14] and dog [15]. However, the major drawback with these models is the long timescale for OA development, which can make studies prohibitively expensive and ultimately unfeasible. Furthermore, public resistance to the use of companion animal species in biomedical research also presents additional challenges.

In contrast to the above models, commercial pigs have been reported to develop spontaneous joint pathologies at a young age, resulting in pigs often being slaughtered due to lameness [16]. However, no study had previously examined whether the commercial pig develops signs of OA joint damage. The aim of this study was two-fold. Firstly, to assess pain-associated behaviour and femorotibial joint pathology for signs of spontaneous development of OA in commercial pigs from juvenile to older adult. Secondly, to assess the potential utility of porcine cartilage explants and isolated porcine chondrocytes and osteoblasts for in vitro and ex-vivo preclinical studies.

\section{Methods}

\section{Animals}

Female pigs (Large white $\mathrm{x}$ Landrace $\mathrm{x}$ Duroc) aged 610 wks $(n=8$, weight $37.4 \pm 1.4 \mathrm{~kg}), 17 \mathrm{wks}$ ( $n=6$, weight $84.0 \pm 2.2 \mathrm{~kg}), 40$ wks $(\mathrm{n}=6$, weight $141.2 \pm 4.2 \mathrm{~kg}), 63$ wks $(\mathrm{n}=8$, weight $245.1 \pm 9.0 \mathrm{~kg})$, and $3-4$ years $(n=7$, weight $230.6 \pm 9.0 \mathrm{~kg}$ ) purchased from JSR Genetics Ltd. (Driffield, UK) were used in this pilot study to examine the incidence of the development of osteoarthritis. For comparison purposes animals were characterised by age in to three groups: juvenile (age range 6-17 wk), adult (40-80 wk), and older adult (3-4 years). The juvenile and adult age groups were all gilts (female pigs that have not been used for breeding), whilst the older adults were ex-breeding sows. Ethical permission for the study was granted by the University of Nottingham Animal Welfare Ethical Review Body (AWERB). Pigs were group-housed under directives set by the Department of Environment, Food, and Rural Affairs (DEFRA), as specified in The Welfare of Farmed Animals (England) Regulations 2007, thus replicating the standards animals were housed in prior to arrival at our facilities. Animals were checked daily by qualified animal technicians. Pigs were provided with free access to food and water and allowed to acclimatise to their surroundings upon arrival at the University facilities for a minimum of 2 weeks before being slaughtered by electrical stunning followed by exsanguination. The 63 wk. animals were maintained for an extended period of time (16-17 weeks), prior to euthanasia, to allow temporal changes in animal behaviour to be assessed. In all animals, after death had been confirmed, the stifles were removed for examination and tissue collection.

\section{Assessment of pain-associated behaviour}

Different aspects of pig behaviour including lameness, response to touch, willingness to ambulate and vocalisation were assessed as potential markers of behavioural pain in juvenile, adult and older adult pigs. Each behavioural aspect was incorporated into a scoring system and assigned a value from 1 to 5 (Additional file 1: Table S1) based on that used by Royal et al. [17]. Behavioural assessment was carried out weekly and assessment sessions typically lasted about an hour. On arrival at the facility, pigs were allowed to acclimatise to the presence of the scorer for between 5 and $10 \mathrm{~min}$ before behavioural scoring was carried out. Observation of lameness was carried out during weekly weighing procedures, with animals encouraged to move by the animal technicians. Similarly, the response to handling by the animal technician during the weighing process was used to record the response to touch. All other scoring parameters were collected on the same visit and prior to attempts to weight the animals, with animals resting in their home environment.

\section{Chondropathy scoring}

Femoral condyles and tibial plateaus of juvenile, adult, and older adult porcine femorotibial joints were used for chondropathy scoring. The lateral and medial surfaces of the femoral condyles and tibial plateaus of the joint were scored separately. Chondropathy scoring was performed using two methodologies, namely Collin's grading and the revised Système Française D'Arthroscopie (SFA) scoring method as described by Walsh and colleagues 
[18]. The Collin's grading and SFA are macroscopic severity scoring systems, which are based on an assessment of OA changes in the articular surface, including cartilage swelling, fibrillation and exposure of bone. They have been widely validated in both mild and severe OA [18-23]. As a comparison to human OA, the femoral condyles and tibial plateau were also scored from end-stage knee OA patients $(n=4)$, which were collected from the Royal Orthopaedic Hospital (Birmingham) following ethical approval from the Research Ethics Committee (NRES 13/NE/0222). In subsequent experiments, based on gross assessment, "damaged cartilage" was defined as having evidence of fibrillation, equivalent to a Collin's grade score of greater than 2, or a revised SFA score of more than 20. Cartilage defined as "undamaged" had a normal, unbroken surface.

\section{Safranin-O staining of femoral condyle proteoglycans}

Femoral condyles from $n=8$ adult animals were snap frozen in liquid nitrogen immediately following slaughter and stored at $-80^{\circ} \mathrm{C}$ until cryostat processing. Cryosections $(8 \mu \mathrm{m}$ thick) were cut using a cryostat and transferred to slides to facilitate subsequent safranin-O/fast green staining. Staining was performed without fixation as described previously [24].

\section{Culture of primary porcine chondrocytes, osteoblasts and cartilage explant}

Primary porcine chondrocytes were isolated from juvenile ( $n=6$ animals) and older adult ( $n=6$ animals) femoral condyle cartilage by collagenase digestion. In brief, samples of cartilage were diced with a scalpel and digested for $4 \mathrm{~h}$ in chondrocyte cell culture media (DMEM supplemented with 10\% FBS, 2 mM L-glutamine, $1 \%$ non-essential amino acids, $1 \%$ penicillin/streptomycin) containing $2 \mathrm{mg} / \mathrm{ml}$ sterile-filtered collagenase (Sigma Aldrich, Poole, UK). The digested cartilage was filtered through a sterile $40 \mu \mathrm{m}$ cell strainer, placed in T75 culture flasks with cell culture media and incubated at $37^{\circ} \mathrm{C}, 5 \%$ $\mathrm{CO}_{2}$. Media was refreshed every $3-4$ days.

Osteoblasts were cultured out from subchondral bone chips obtained from adult $(n=6)$ and older adult $(n=6)$ pigs, based on a protocol we have previously used for human OA subchondral bone osteoblast outgrowth [25]. In brief, subchondral bone chips from damaged and nondamaged porcine femoral condyles were incubated in T75 culture flasks at $37^{\circ} \mathrm{C}, 5 \% \mathrm{CO}_{2}$ in osteoblast cell culture media (DMEM supplemented with 10\% FBS, $2 \mathrm{mM}$ L-glutamine, $1 \%$ non-essential amino acids, $1 \%$ penicillin/ streptomycin, $2 \mathrm{mM} \beta$-glycerophosphate, $50 \mu \mathrm{g} / \mathrm{ml} \mathrm{L-ascor-}$ bic acid, $10 \mathrm{nM}$ dexamethasone and $1 \%$ amphotericin-B). After 7-20 days of culture, osteoblast outgrowth was observed and the bone chips were removed.
Cartilage explants were prepared using a cork borer to cut cartilage discs $(50 \mathrm{~mm}$ diameter) from full thickness sections of damaged and non-damaged femoral condyle cartilage obtained from adult animals $(n=7)$ for sGAG analysis. Cartilage explants were also prepared from older adult animals $(n=5)$ for analysis of collagen mRNA and for in vitro cytokine stimulation. Explant discs were placed into 96-well cell culture plates in chondrocyte cell culture media and incubated at $37^{\circ} \mathrm{C}, 5 \% \mathrm{CO}_{2}$.

\section{1,9-dimethylmethylene blue (DMMB) proteoglycan release assay}

Sulphated glycosaminoglycan (sGAG) released from cartilage explant ( $\mathrm{n}=7$ adult animals) was quantified via a dimethylmethylene blue (DMMB) assay, as previously described [26]. In brief, cartilage explants were allowed to rest in chondrocyte media for 2-3 days before being replaced with fresh chondrocyte media and incubated for $48 \mathrm{~h}$, after which time the supernatant was collected for analysis. Shark chondroiten sulphate C (Sigma, UK) was used to generate a standard curve and $40 \mu \mathrm{L}$ of standards and samples combined with $250 \mu \mathrm{L}$ DMMB reagent ( $0.24 \%$ sodium chloride, $0.3 \%$ glycine, $0.8 \% \mathrm{v} / \mathrm{v}$ hydrochloric acid, 0.0016\% DMMB) and absorbance read at $550 \mathrm{~nm}$ using a microplate reader (Bio-Rad 680XR).

\section{Quantification of mRNA expression by qRT-PCR}

Total RNA was extracted from primary porcine chondrocytes using an RNA isolation kit (Roche High Pure Isolation Kit) according to the manufacturer's instructions. Total RNA was extracted from snap-frozen porcine femoral condyle cartilage tissue using ceramic beads (Roche Green Beads) and a MagnaLyser instrument in combination with a fibrous tissue RNA extraction kit (Qiagen). cDNA was subsequently generated from $100 \mathrm{ng}$ of total RNA (ReverAid RT cDNA synthesis kit, Thermo Scientific) according to manufacturer's instructions. Relative mRNA expression of the collagen genes COL1A1 and COL2A1 were determined by qRTPCR using a Roche Lightcycler $480^{\circ}$ (Roche, Burgess Hill, UK), normalised to cDNA concentration. The primer sequences used were as follows: COL1A1 Forward: AGAAGAAGA CATCCCACCAGTCA, Reverse: CGTCATCGCACAAC ACATTG; COL2A1 Forward: GGCAACAGCAGGTTCA CGTA, Reverse: CAATCATAGTCTGGCCCCACTT. All samples were analysed in triplicate.

\section{Alkaline phosphatase (ALP) assay}

Cultured osteoblasts were lysed using cell lysis buffer (150 mM Sodium Chloride, $1 \%$ triton $\mathrm{x}-100,50 \mathrm{mM}$ Tris, $\mathrm{pH}$ 8.0) containing protease and phosphatase inhibitor cocktails (Sigma Aldrich, Poole, UK) and the protein concentration of cell extract determined using the Bradford protein assay [27]. To $10 \mu \mathrm{l}$ osteoblast lysate, 
$100 \mu \mathrm{l}$ of alkaline phosphatase substrate containing $p$-nitrophenylphosphate (pNNP) was added and incubated for 15 mins at $37^{\circ} \mathrm{C}$ before being stopped by the addition of $20 \mu \mathrm{l}$ $0.1 \mathrm{M}$ sodium hydroxide. Standards prepared from human alkaline phosphatase diluted in $1 \mathrm{mM}$ magnesium chloride solution were run in parallel. Absorbance was measured at $405 \mathrm{~nm}$ using a BioRad 680XR platereader.

\section{Alizarin red mineralisation assay}

Mineralisation of osteoblasts was determined by Alizarin Red staining [28]. In brief, upon reaching confluence osteoblasts were grown for a further 3 weeks and then were stained with $0.5 \%$ alizarin red staining solution $(0.5 \%$ Alizarin Red, 1\% ammonia solution, $\mathrm{pH} 4.0$ ) for $10 \mathrm{~min}$. The cells were then washed in PBS and destained using $10 \%$ cetyl pryridium chloride (Sigma, UK) for $10 \mathrm{~min}$. The absorbance of the supernatant was measured at $550 \mathrm{~nm}$ on a BioRad 680XR platereader.

\section{Mitochondrial assays}

To isolate mitochondria, osteoblasts were resuspended in $2 \mathrm{ml}$ Buffer $(100 \mathrm{mM}$ potassium chloride, $50 \mathrm{mM}$ Tris, $5 \mathrm{mM}$ Magnesium Chloride, $1.8 \mathrm{mM}$ ATP, $1 \mathrm{mM}$ EDTA. $\mathrm{pH}$ 7.2) and homogenised on ice for $4 \mathrm{~min}$. The sample was then centrifuged at $720 \mathrm{x}$ g for $1 \mathrm{~min}$ to pellet any cellular debris. The resultant supernatant was transferred to clean pre-cooled tube and centrifuged at 10000 $\mathrm{x} \mathrm{g}$ for $5 \mathrm{~min}$ to pellet the mitochondria. The mitochondrial pellet was then resuspended in $400 \mu \mathrm{l}$ buffer (225 $\mathrm{mM}$ sucrose, $44 \mathrm{mM}$ potassium phosphate monobasic, $12.5 \mathrm{mM}$ magnesium acetate, $6 \mathrm{mM}$ EDTA). Maximal mitochondrial ATP production was measured using a 96-well bioluminescence assay. In brief, $25 \mu \mathrm{l}$ of the mitochondrial sample was added to $110 \mu \mathrm{l}$ Tris-EDTA buffer, $25 \mu \mathrm{l} \mathrm{ADP}$ and $40 \mu \mathrm{l}$ ATP reagent SL (Biothema ATP Reagent SL Kit). All samples were run in triplicate and luminescence measured using a FLUOstar plate reader. Mitochondrial citrate synthase activity was measured based on the kinetic production of 2-nitro-5-benzoic acid measured at an absorbance of $415 \mathrm{~nm}$ using a Bio-rad 680XR microplate reader.

\section{Statistical analysis}

Statistical analysis was carried out using Graphpad Prism software version 7.0. Chondropathy scores and lameness scores were analysed using the Kruskall-Wallis non-parametric test, with post-hoc tests where appropriate. Pearson's correlation coefficient was used to determine the relationship between proteoglycan loss and revised SFA chondropathy score. In vitro and ex-vivo expression data was analysed using unpaired t-tests with 1-way ANOVA used for dose responses.

\section{Results}

Development of lameness as a marker of pain-associated behaviour in the commercial pig

Lameness was assessed in pigs at three different age groups, (i) juvenile $(n=6)$, (ii) adult 63 week old pigs $(n=6)$ and (iii) older adults $(n=7)$. The adult pigs (63 week old) were monitored at weekly intervals over a 16 week period and lameness assessed from age 64 to 80 weeks, to allow temporal changes in pain-associated behaviour to be observed. Observational scoring of lameness indicators revealed no indications of lameness in the youngest age group (juvenile). However, there was a significant increase $(p<0.05)$ in the median lameness of both 80 week adult and older adult pigs, compared to juvenile pigs (Fig. 1a). Furthermore, there was a significant $(p<0.01)$ increase in lameness score over a timespan of 16 weeks in adult pigs from age 64 to 80 weeks old (Fig. 1b). Lameness in the older adult animals was on average no greater than that observed in adult animals aged 80 wks (Fig. 1a). None of the animals responded to touch in a manner that would be associated with pain or discomfort, or differed in their willingness to ambulate. Furthermore, vocalisation was not related to any pain or discomfort as might be expected of other animals, such as the rat [29] for which VAS scoring is more commonly used.

\section{The commercial pig spontaneously develops signs of osteoarthritic joint damage}

Femorotibial joints from juvenile, adult, and older adult commercial pigs were assessed for the presence and severity of OA lesions by chondropathy scoring using Collin's grading and a revised SFA scoring system. As a comparison to human OA, femoral condyles and tibial plateau from end-stage human knee OA patients $(n=4)$ were scored using the same chondropathy scoring system.

Chondropathy scoring showed an effect of age and joint compartment on the development of joint damage in the commercial pig. Using either Collin's grading or revised SFA showed a significant increase in the median joint damage score in adult and older adult pigs, compared to juvenile pigs (Fig. 2a, b). As expected, the greatest joint damage was observed in the older adult pigs, which exhibited grade II and grade III lesions in femoral condyle cartilage (Fig. 2c). OA cartilage lesions developed on both tibial plateaus and femoral condyles, and on both medial and lateral sides of the joint. However, in the older adult pigs significantly greater median joint damage was present on the medial side of the femoral condyles and on the medial side of tibial plateau, compared to the corresponding lateral compartments $(p<0.05)$ as scored using revised SFA (Fig. 2a). In addition to cartilage lesions, the joints of all older adult pigs animals, and the majority (75\%) of 80 week adult pigs exhibited bony nodules, indicative of osteophyte formation (Fig. 2d). However, notably, joint damage, even in the older adult pigs was lower than 


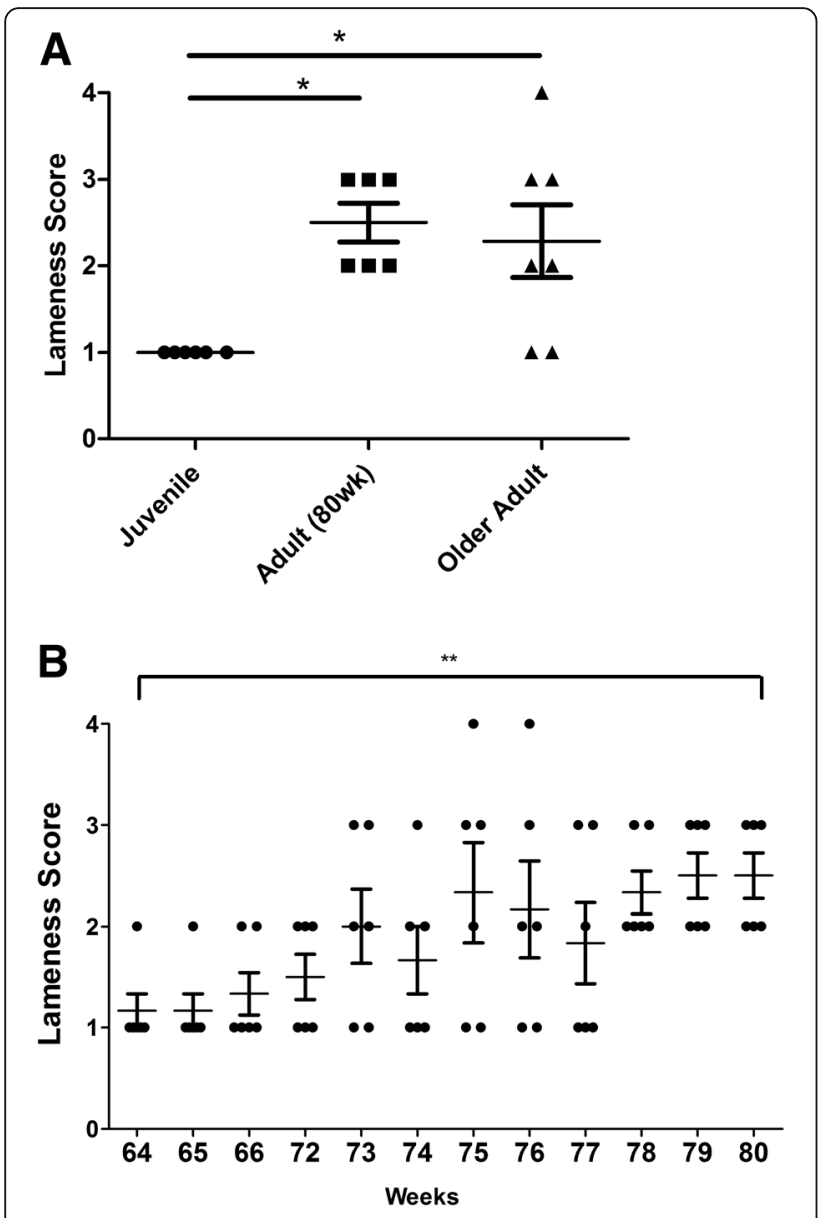

Fig. 1 Development of lameness as a marker of behavioural pain. A scoring system was used to assess lameness as a marker of behavioural pain. a Comparison of median lameness score in juvenile ( $n=6$ animals), 80 wk. adult ( $n=6$ animals) and older adult ( $n=7$ animals) pigs. ${ }^{*}=p<0.05$, significantly different from juvenile lameness score as determined by Kruskal-Wallis non-parametric test with Dunn's post-hoc test. $\mathbf{b}$ Median lameness score in adult pigs across an 16 week timespan from age 64 to $80 \mathrm{wk}$. old ( $n=6$ animals). ${ }^{* *}=p<0.01$, significant change in median score over time as determined by Kruskal-Wallis test

that observed in end-stage human knee OA (Collin's grade $=10.0 \pm 1.1$; SFA $=106.4 \pm 10.8$ ) .

In order to further examine the pathology of these lesions we prepared cryosections of femoral condyle cartilage from $n=8$ adult pigs which exhibited varying degrees of joint damage, and stained the cartilage proteoglycans with Safranin O. As expected, proteoglycan staining was appreciably lower in the cartilage sections prepared from pig joints that exhibited higher chondropathy scores (Fig. 3a). We then assessed the relationship between proteoglycan degradation and joint damage by preparing cartilage explants from $n=7$ adult pigs with varying degrees of joint damage (femoral condyle SFA = 1.4, 2.1, 3.2, 7.6, 11.2, 13.4 and 30.5) and measuring the release of sGAGs, compared to non-damaged cartilage explant. Due to the requirement to obtain full thickness cartilage explants of the same size $(50 \mathrm{~mm}$ diameter) we did not determine sGAG release from explants of joints with higher chondropathy scores. Therefore, a limitation is that we have not assessed the relationship between cartilage explant sGAG release across the full range of joint damage scores in these animals. Nevertheless, the relative release of sGAGs was positively correlated with both Collin's grade $\left(\mathrm{r}^{2}=0.791, p<0.01\right)$ and revised SFA score $\left(r^{2}=0.733, p<0.05\right)$ (Fig. 3b).

\section{Cytokine stimulation of primary porcine chondrocytes and cartilage explant induces IL- 6 release and proteoglycan degradation}

Since human OA cartilage degeneration has been attributed to the hypertrophy and increased proliferative activity of chondrocytes, we first examined whether chondrocytes from the damaged joints of older adult pigs exhibited a greater proliferative capacity. Comparing primary porcine chondrocytes isolated from older adult pigs with those isolated from juvenile animals, there was no difference in proliferation rate (Fig. 4a). However, similar to primary human chondrocytes, upon 2D culture porcine primary cells rapidly adopted a fibroblast-like morphology (Fig. 4b) and exhibited a significantly lower ratio of COL2A1 to COL1A1 expression compared to cartilage (Fig. 4c; $p<0.05$ ), suggesting that the porcine chondrocyte phenotype is not maintained in culture.

Next in porcine femoral condyle cartilage explants and isolated porcine chondrocytes obtained from older adult animals, we examined their utility as ex-vivo and in vitro OA models by determining the effect of putative pro-inflammatory drivers of OA cartilage degeneration on the release of the pro-inflammatory cytokine IL-6 (by ELISA) and sGAG release (by DMMB assay). Stimulation for $24 \mathrm{~h}$ of primary porcine chondrocytes with either recombinant porcine IL-1 $\beta(0.1 \mathrm{ng} / \mathrm{ml}$ to $3 \mathrm{ng} / \mathrm{ml})$ or recombinant visfatin $(500 \mathrm{ng} / \mathrm{ml})$ significantly increased IL-6 secretion, compared to unstimulated control (Fig. 4d). Similarly, 24h stimulation of porcine cartilage explants with either recombinant IL-1 $(0.1 \mathrm{ng} / \mathrm{ml}$ to $1 \mathrm{ng} / \mathrm{ml})$ or visfatin $(500 \mathrm{ng} / \mathrm{ml})$ significantly induced IL-6 secretion (Fig. 4e), compared to unstimulated control cells. In addition, $24 \mathrm{~h}$ stimulation of porcine cartilage explant with IL- $1 \beta$ significantly induced the release of sGAGs (Fig. 4f).

Porcine subchondral osteoblasts from OA damaged joints exhibit reduced proliferative and metabolic capacities and reduced ability to mineralise

The presence of bony nodules in adult animals indicated the involvement of bone in the development of joint problems in the pig. In human OA, the presence of osteophytes and changes to the subchondral bone trabecular structure has been attributed to an altered osteoblast phenotype [30]. 

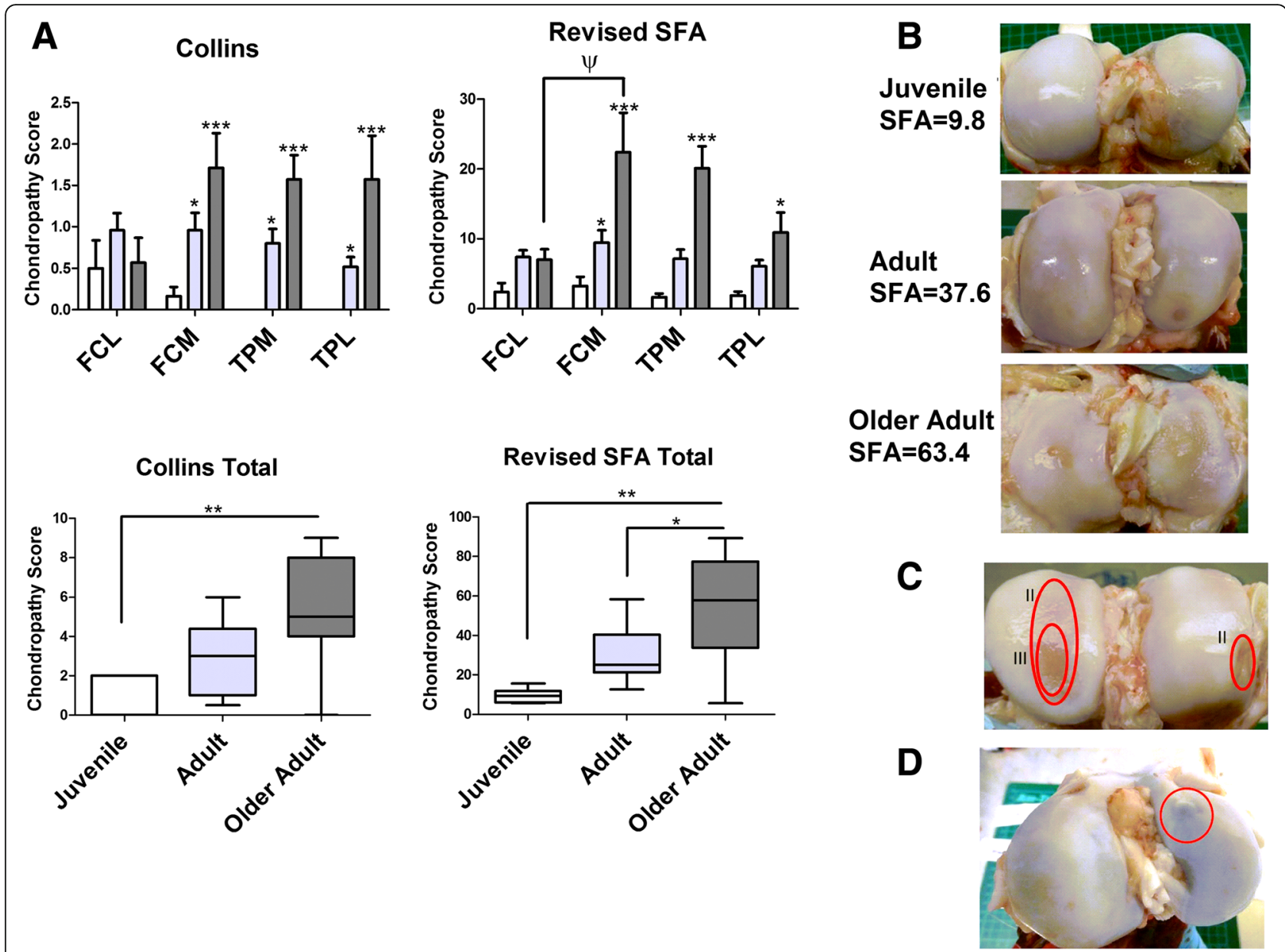

Fig. 2 Spontaneous development of joint damage in the commercial pig. a Median total and joint compartment chondropathy score of femoral condyle and tibial plateau joints using Collin's grading and Revised SFA in juvenile (white boxes, $n=6$ ), adult (light grey boxes, $n=14$ ) and older adult (dark grey boxes, $n=7$ ) pigs. ${ }^{*}=p<0.05 ;{ }^{* *}=p<0.01 ; * *=p<0.001$ significantly different compared to juvenile animals. $\psi=p<0.05$ significantly different between medial and lateral compartment within same age group, as determined using Kruskal-Wallis non-parametric test. b Representative images of femoral condyle joints from juvenile, adult, and older adult animals. c Evidence of Grade II and Grade III cartilage lesions in femoral condyles of older adult pigs $\mathbf{d}$ Evidence of bony nodules in adult pigs. FCM = femoral condyle medial, $\mathrm{FCL}=$ femoral condyle lateral, $\mathrm{TPM}=$ tibital plateau medial, $\mathrm{TPL}=$ tibial plateau lateral

Therefore, we next compared the phenotype of osteoblasts isolated from both damaged and non-damaged joints from both adult and older adult pigs.

The proliferative rate of osteoblasts obtained from non-damaged adult joints was significantly $(p<0.01)$ greater than in osteoblasts from the older adult damaged joints (Fig. 5a). In addition, the osteoblasts from non-damaged adult joints, but not osteoblasts from the damaged older adult joints, were able to form mineralised bone nodules over $21 \mathrm{~d}$ culture as noted by positive staining of mineral with Alizarin red (Fig. 5b). Furthermore, while not statistically significant, a trend for osteoblasts from damaged older adult joints to exhibit greater ALP activity than osteoblasts from adult non-damaged joints was observed ( $P=0.09$; Fig. $5 \mathrm{c}$ ).

To investigate this further, we next examined mitochondrial activity by determining both mitochondrial ATP production and citrate synthase activity in osteoblasts from damaged and non-damaged joints collected either adult or older adult pigs. There was a highly significant $(p<0.01)$ effect of joint damage on both mitochondrial ATP production (Fig. 5d) and citrate synthase activity (Fig. 5e), with osteoblasts from damaged joints exhibiting reduced mitochondrial ATP production and citrate synthase activity, compared to osteoblasts from non-damaged joints, in both adult and older adult pigs.

\section{Discussion}

This study is the first to report the temporal and spontaneous development of $\mathrm{OA}$ in the femorotibial joint of the commercial pig and its association with behavioural pain. Furthermore, for the first time we provide evidence of impaired proliferative, metabolic and mineralisation capacity of subchondral osteoblasts from OA damaged joints in the pig. 


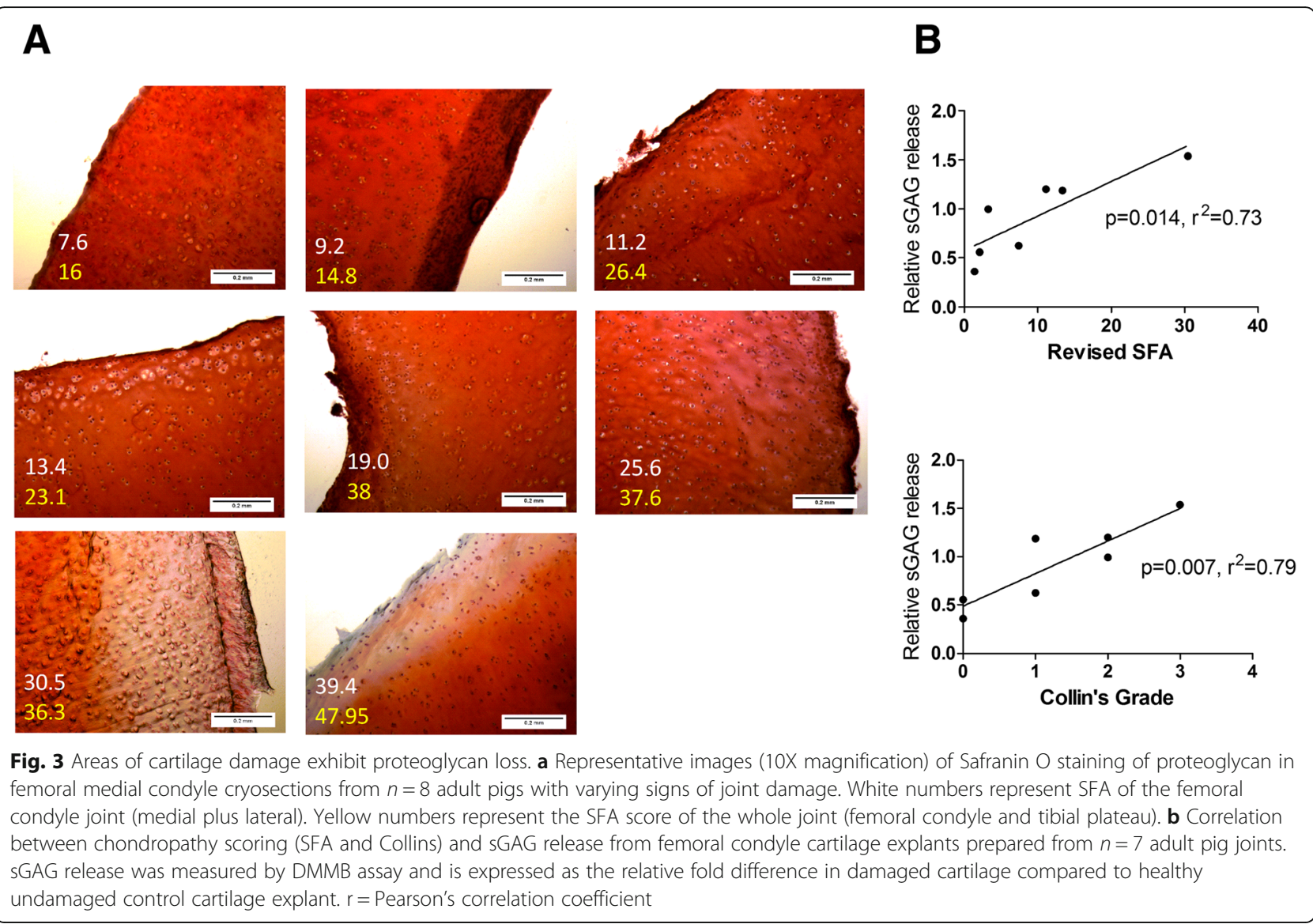

Examination of porcine femorotibial joints by two different chondropathy scoring systems (revised SFA and Collin's grade) revealed that commercial pigs develop osteoarthritic lesions in their femorotibial joints early in their lifespan. Importantly, significantly greater total cartilage damage was observed on the medial side of the joint compared to the lateral compartments in the older adult pigs, as often observed in the development of human OA knee which has been attributed to gait [10].

Similarly to human OA, articular cartilage from damaged pig joints exhibited lower proteoglycan content than that collected from non-damaged joints. In human $\mathrm{OA}$, cartilage proteoglycan matrix degradation is attributed to the proliferation and hypertrophy of chondrocytes. In this study, we observed no difference in the proliferative capacity of porcine primary chondrocytes isolated from juvenile joints compared to chondrocytes isolated from damaged older adult pig joints. However, similarly to human OA chondrocytes [31-33] porcine chondrocytes rapidly de-differentiated in culture, exhibiting a fibroblast-like morphology and expressed significantly lower type II collagen compared to porcine cartilage.

In addition to cartilage damage, the joints of adult pigs exhibited bony nodules indicative of osteophyte formation and aberrant subchondral bone remodelling [34]. Evidence of abnormalities in the subchondral bone early in the development of joint damage in the pig adds further weight to the role of bone in the initiation and progression of OA $[2,35,36]$. In human OA, the appearance of bony spurs can be seen on radiographs of the diseased joint, whilst MRI analysis has shown that the presence of bone marrow lesions in subchondral bone are associated with the progression of cartilage loss [37, 38] and pain [39, 40]. Further analysis by MicroCT has shown that the subchondral bone in $\mathrm{OA}$ is under-mineralised but has thicker trabeculae [41], suggestive of accelerated bone turnover. In addition to our findings in the pig, changes to the subchondral bone have been reported during the spontaneous development of OA in the Dunkin Harltey guinea pig [42], where bone changes were found to occur prior to significant cartilage loss [43].

In this study, further evidence for the involvement of bone in the spontaneous development of OA in the pig was observed upon analysis of isolated porcine osteoblasts from the subchondral bone tissue of damaged joints and non-damaged joints. Both proliferative and mineralisation capacity was found to be impaired in osteoblasts from damaged joints of older adult animals compared to osteoblasts from younger non-damaged joints. Conversely, osteoblasts 


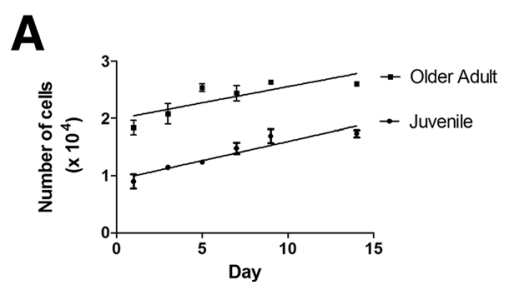

B

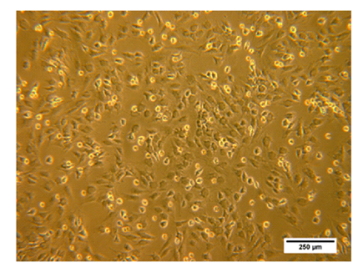

\section{C}
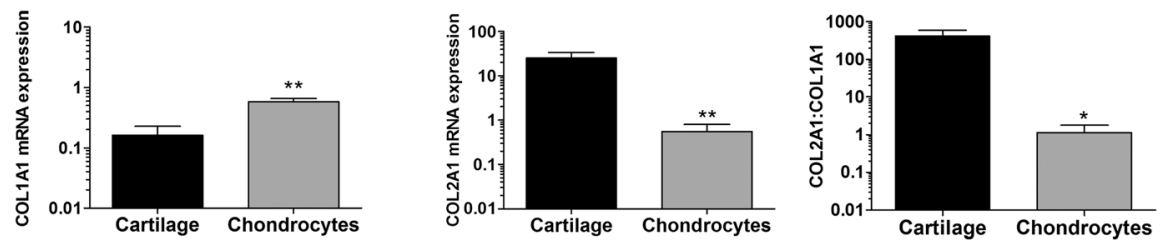

D

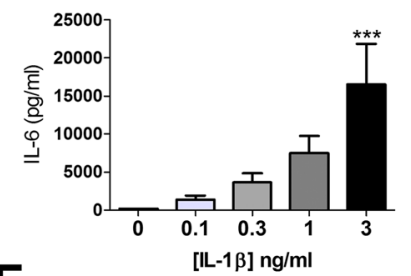

E
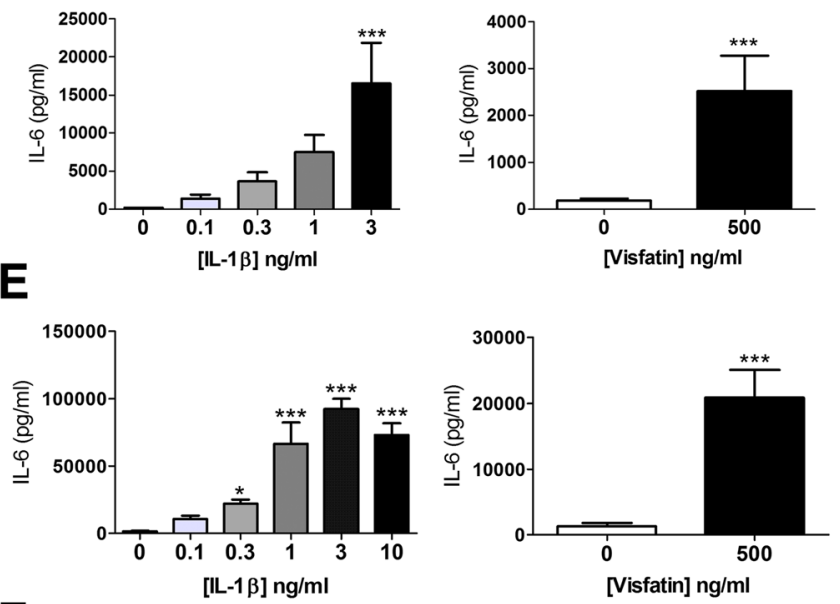

E

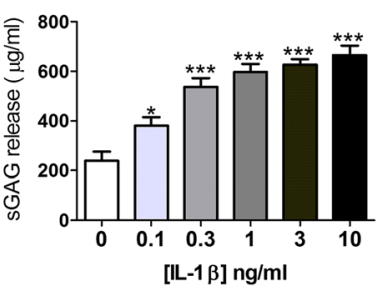

Fig. 4 Characterisation of porcine chondrocytes and cartilage explant. a Proliferation of primary porcine chondrocytes isolated from juvenile $(n=6)$ and older adult pigs $(n=6)$. Proliferation was determined by MTS assay over a timecourse of 14 days. (B) Representative light microscope image (6.3X magnification) of porcine chondrocytes in 2D culture showing fibroblast-like morphology. $\mathbf{c}$ mRNA expression of type I and Type II collagen in primary porcine chondrocytes ( $n=6$ animals) compared to non-damaged porcine cartilage explant $(n=5$ animals), from older adult pigs. Expression was determined by qRT-PCR normalised to total CDNA concentration. $\mathbf{d}$ Secretion of IL-6 from porcine primary chondrocytes from older adult pigs $(n=6)$ stimulated for $24 \mathrm{~h}$ with recombinant IL-1 $\beta(0.1-3 \mathrm{ng} / \mathrm{ml})$ or recombinant visfatin $(500 \mathrm{ng} / \mathrm{ml})$. IL-6 in cell supernatants was measured by ELISA. ${ }^{*}=p<0.05 ;{ }^{* * *}=p<0.001$ significantly different from un-stimulated control chondrocytes. Bars represent mean \pm SEM $(n=6)$. e Secretion of IL-6 from porcine non-damaged cartilage explants from older adult pigs stimulated for $24 \mathrm{~h}$ with recombinant IL-1ß $(0.1-10 \mathrm{ng} / \mathrm{ml})$ or recombinant visfatin $(500 \mathrm{mg} / \mathrm{ml})$ as measured by ELISA. ${ }^{*}=p<0.05 ;{ }^{* * *}=p<0.001$ significantly different from un-stimulated control explants. Bars represent mean \pm SEM ( $n=20$ explants per stimulant). f Detection of sulphated glycosaminoglycan (sGAG) proteoglycan side-chain upon $24 \mathrm{~h}$ stimulation of porcine non-damaged cartilage explant from older adult pigs with recombinant IL-1B. sGAG detected by DMMB assay. ${ }^{*}=p<0.05 ; * * *$ $=p<0.001$ significantly different from un-stimulated control explants. Bars represent mean $\pm \operatorname{SEM}(n=20$ explants per stimulant)

from damaged joints of older adult animals exhibited greater ALP activity compared to osteoblasts from younger non-damaged joints. It is important to note however, that we did not compare the proliferative activity of osteoblasts from damaged and non-damaged joints within the same age group. We cannot therefore be certain whether this impaired proliferative osteoblast phenotype is due to age or disease. However, this "damaged" porcine osteoblast 


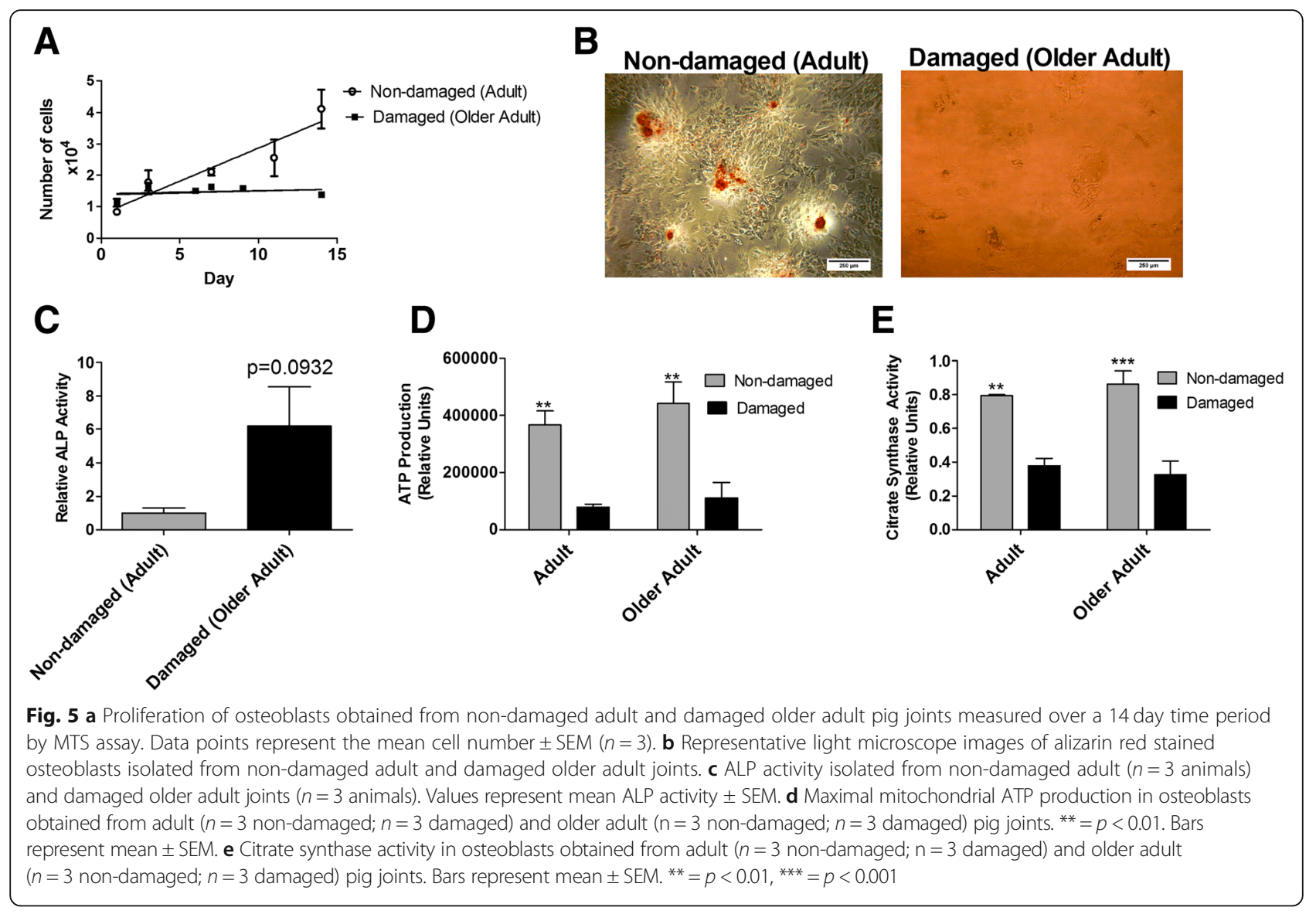

phenotype has similarities to the human OA osteoblast phenotype. For example, human OA subchondral osteoblasts have elevated ALP activity, compared to non-OA osteoblasts [44]. Furthermore, Sanchez et al. [45] have found that osteoblasts from regions of sclerotic subchondral bone tissue exhibit greater ALP activity and reduced mineralization, compared to non-sclerotic osteoblasts from OA joints. This high ALP activity but lower mineralisation capacity in OA osteoblasts has been attributed to the potential accumulation of pyrophosphate (PPi) activating ALP, whilst being a potent inhibitor of hydroxyapaptite crystal formation [45].

It has previously been proposed that the phenotype of elevated ALP coupled with reduced mineralisation in OA osteoblasts indicates that subchondral bone osteoblasts undergo incomplete differentiation in human OA [46]. Since mitochondrial activity plays a critical role in osteoblast differentiation $[47,48]$ it is notable that we found that osteoblasts from damaged pig joints exhibited significantly lower mitochondrial ATP production than osteoblasts from non-damaged joints. Mitochondrial activity has been implicated as a mediator of OA pathology [49, 50]. Indeed, reduced mitochondrial activity in OA chondrocytes has been implicated in cartilage damage [51]. Currently, despite mitochondrial activity being central to osteoblast differentiation, studies investigating mitochondria in OA osteoblasts are lacking. However, osteoblast mitochondrial dysfunction has been identified as an important factor in the pathogenesis of osteoporosis [52]. Our finding that citrate synthase activity was also reduced in osteoblasts from damaged pig joints suggests joint damage in the pig was associated with a reduction in subchondral osteoblast mitochondrial number, rather than osteoblast mitochondrial dysfunction.

In addition to the spontaneous development of OA joint damage, the commercial pig represents an excellent tissue source for in vitro and ex-vivo OA models. Indeed, cultured osteoblasts retained a "damaged" dysfunctional phenotype in vitro. Furthermore, although isolated chondrocytes rapidly de-differentiated upon 2D culture, both chondrocytes and cartilage explants were highly responsive to stimulation with pro-inflammatory putative OA drivers including IL-1 $\beta$ and visfatin, with a rapid quantitative secretion of IL-6, and sGAG release. Critically, such studies on human cartilage most often utilise tissue from end-stage OA patients where the cartilage is highly degraded. Therefore, the availability of sufficient quantity of relatively healthy cartilage tissue that behaves in a similar manner may represent a useful tissue source for conducting studies that better represent early OA initiation. 
The use of the commercial pig as a spontaneous model of OA has some limitations. Pigs can develop osteochondrosis (OC), which commonly occurs in fast growing animals and can lead to the development of OA which is secondary [53]. Also, while the Large white $x$ Landrace $x$ Duroc, as used in the current study, is commonly utilized for commercial purposes, it is feasible that differences in the development of OA could be seen between different genotypes of pig. A limitation of the current study is the narrow range of recombinant proteins considered in our ex vivo assays. The response of cartilage explants from the pig femorotibial joint to alternative proteins implicated in the etiology of human osteoarthritis remains to be confirmed. Furthermore, the cytokine stimulations of porcine chondrocytes and cartilage explant were conducted in full serum culture media. This was done in order to mimic previous studies in on cytokine stimulation of human cartilage and human chondrocytes [54, 55]. However, it should be noted that Bian et al. showed that cartilage explant cultured in full-serum exhibited $70 \%$ greater degradation over the course of 2 weeks compared to cartilage in serum-free media [56]. Finally, it remains to be determined whether histological examination of the subchondral bone of the pig femorotibial joint will reveal similar pathophysiological changes as evident in humans.

\section{Conclusion}

The commercial pig spontaneously develops behavioural pain and OA joint damage in the femorotibial joint with evidence of cartilage lesions in the femoral condyles and tibial plateau and metabolically dysfunctional subchondral bone osteoblasts. The commercial pig may therefore provide an alternative preclinical model of OA and a highly useful source of joint tissue for in vitro and ex vivo OA models.

\section{Additional file}

\section{Additional file 1: Table S1. Scoring of behavioural pain aspects.} (DOCX $14 \mathrm{~kb})$

\section{Abbreviations \\ ALP: Alkaline Phosphatase; AWERB: Animal Welfare Ethical Review Body; DMMB: 1,9-dimethylmethylene blue; DMOADs: disease modifying OA drugs; FCL: Femoral condoyle - lateral surface; FCM: Femoral condoyle - medial surface; OA: Osteoarthritis; pNNP: p-nitrophenylphosphate; SFA: Système Française D'Arthroscopie; sGAG: Sulphated glycosaminoglycan; TPL: Tibial plateau - lateral surface; TPM: Tibial plateau - medial surface}

\section{Acknowledgments}

The authors would like to thank Kirsty Jewell (University of Nottingham) for technical support.

\section{Funding}

Mhairi Macfadyen was supported by a MRC-ARUK Centre for Musculoskeletal Ageing Research PhD studentship (MR/K00414X/1). The funding agency had no input in to the design of the study, the collection, analysis and interpretation of data, the writing of the manuscript, or the decision to publish.

\section{Availability of data and materials}

The datasets supporting the conclusions of this article are included within the article. If you wish to obtain access for the underlying material please contact the corresponding author to discuss your request in detail.

\section{Authors' contributions}

MM performed data collection, analysis of data and drafted the manuscript. ZD performed data collection, analysis of data. SK performed data collection and analysis of data. TP, JMB, AJM and SWJ conceived and designed the study, analysed the data and wrote the manuscript. All authors approved the final manuscript.

\section{Ethics approval and consent to participate}

This study was approved by the University of Nottingham Animal Welfare Ethical Review Body (AWERB). The collection and use of human OA joint tissue was approved by the National Research Ethics Committee (NRES 13/NE/0222) and written informed consent was obtained from patients.

\section{Consent for publication}

Not applicable.

\section{Competing interests}

Dr. Simon Jones is a member of the Editorial Board of BMC Musculoskeletal Disorders. The authors declare that they have no other competing interests.

\section{Publisher's Note}

Springer Nature remains neutral with regard to jurisdictional claims in published maps and institutional affiliations.

\section{Author details}

${ }^{1}$ MRC-ARUK Centre for Musculoskeletal Ageing Research, School of Biosciences, University of Nottingham, Sutton Bonington, UK. ${ }^{2}$ Metabolism Unit, Shriners Hospitals for Children, Galveston, TX, USA. ${ }^{3}$ Department of Surgery, University of Texas Medical Branch, Galveston, TX, USA. Institute of Inflammation and Ageing, MRC-ARUK Centre for Musculoskeletal Ageing Research, School of Immunity, University of Birmingham, Birmingham, UK.

Received: 14 June 2018 Accepted: 1 February 2019

Published online: 11 February 2019

\section{References}

1. Murray CJ, Richards MA, Newton JN, Fenton KA, Anderson HR, Atkinson C, Bennett $D$, Bernabe $E$, Blencowe $H$, Bourne $R$, et al. UK health performance: findings of the global burden of disease study 2010. Lancet. 2013;381(9871): 997-1020.

2. Tonge DP, Pearson MJ, Jones SW. The hallmarks of osteoarthritis and the potential to develop personalised disease-modifying pharmacological therapeutics. Osteoarthr Cartil. 2014;22(5):609-21.

3. Philp AM, Davis ET, Jones SW. Developing anti-inflammatory therapeutics for patients with osteoarthritis. Rheumatology (Oxford). 2017;56(6):869-81.

4. Chen A, Gupte C, Akhtar K, Smith P, Cobb J. The global economic cost of osteoarthritis: how the UK compares. Arthritis. 2012;2012:698709.

5. Cross M, Smith E, Hoy D, Nolte S, Ackerman I, Fransen M, Bridgett L, Williams S, Guillemin F, Hill CL, et al. The global burden of hip and knee osteoarthritis: estimates from the global burden of disease 2010 study. Ann Rheum Dis. 2014;73(7):1323-30.

6. Thysen S, Luyten FP, Lories RJ. Targets, models and challenges in osteoarthritis research. Dis Model Mech. 2015;8(1):17-30.

7. Brandt KD, Braunstein EM, Visco DM, O'Connor B, Heck D, Albrecht M. Anterior (cranial) cruciate ligament transection in the dog: a bona fide model of osteoarthritis, not merely of cartilage injury and repair. J Rheumatol. 1991;18(3):436-46.

8. Culley KL, Dragomir CL, Chang J, Wondimu EB, Coico J, Plumb DA, Otero M, Goldring MB. Mouse models of osteoarthritis: surgical model of posttraumatic osteoarthritis induced by destabilization of the medial meniscus. Methods Mol Biol. 2015;1226:143-73.

9. Glasson SS, Blanchet TJ, Morris EA. The surgical destabilization of the medial meniscus (DMM) model of osteoarthritis in the 129/SvEv mouse. Osteoarthr Cartil. 2007;15(9):1061-9.

10. Bendele AM. Animal models of osteoarthritis. J Musculoskelet Neuronal Interact. 2001;1(4):363-76. 
11. Mak IWY, Evaniew N, Ghert M. Lost in translation: animal models and clinical trials in cancer treatment. Am J Transl Res. 2014;6(2):114-8.

12. Jimenez PA, Glasson SS, Trubetskoy OV, Haimes HB. Spontaneous osteoarthritis in Dunkin Hartley Guinea pigs: histologic, radiologic, and biochemical changes. Lab Anim Sci. 1997;47(6):598-601.

13. Tonge DP, Bardsley RG, Parr T, Maciewicz RA, Jones SW. Evidence of changes to skeletal muscle contractile properties during the initiation of disease in the ageing Guinea pig model of osteoarthritis. Longev Healthspan. 2013;2(1):15.

14. Mcllwraith CW, Frisbie DD, Kawcak CE. The horse as a model of naturally occurring osteoarthritis. Bone Joint Res. 2012;1(11):297-309.

15. Liu W, Burton-Wurster N, Glant T, Tashman S, Sumner DR, Kamath RV, Lust G, Kimura JH, Cs-Szabo G. Spontaneous and experimental osteoarthritis in dog: similarities and differences in proteoglycan levels. J Orthop Res. 2003; 21(4):730-7.

16. Jorgensen B, Sorensen MT. Different rearing intensities of gilts: II Effects on subsequent leg weakness and longevity. Livest Prod Sci. 1998;54(2):167-71.

17. Royal JM, Settle TL, Bodo M, Lombardini E, Kent ML, Upp J, Rothwell SW. Assessment of postoperative analgesia after application of ultrasoundguided regional anesthesia for surgery in a swine femoral fracture model. J Am Assoc Lab Anim Sci. 2013;52(3):265-76.

18. Walsh DA, Yousef A, McWilliams DF, Hill R, Hargin E, Wilson D. Evaluation of a photographic Chondropathy score (PCS) for pathological samples in a study of inflammation in tibiofemoral osteoarthritis. Osteoarthr Cartil. 2009; 17(3):304-12.

19. Hopwood B, Tsykin A, Findlay DM, Fazzalari NL. Microarray gene expression profiling of osteoarthritic bone suggests altered bone remodelling, WNT and transforming growth factor-beta/bone morphogenic protein signalling. Arthritis Res Ther. 2007;9(5):R100.

20. Kuettner KE, Cole AA. Cartilage degeneration in different human joints. Osteoarthr Cartil. 2005;13(2):93-103.

21. Ayral X, Gueguen A, Listrat V, Bahuaud J, Beaufils P, Beguin J, Bonvarlet JP, Boyer T, Coudane H, Delaunay C, et al. Proposal of a simplified arthroscopic score of cartilage lesions of the knee (simplified SFA score). Rev Rhum Ed Fr. 1994;61(2):97-9.

22. Dougados M, Ayral X, Listrat V, Gueguen A, Bahuaud J, Beaufils P, Beguin JA, Bonvarlet JP, Boyer T, Coudane $H$, et al. The SFA system for assessing articular cartilage lesions at arthroscopy of the knee. Arthroscopy. 1994; 10(1):69-77.

23. Ravaud P, Giraudeau B, Auleley GR, Chastang C, Poiraudeau S, Ayral X, Dougados M. Radiographic assessment of knee osteoarthritis: reproducibility and sensitivity to change. J Rheumatol. 1996;23(10):1756-64.

24. Schmitz N, Laverty S, Kraus VB, Aigner T. Basic methods in histopathology of joint tissues. Osteoarthr Cartil. 2010;18(Suppl 3):S113-6.

25. Chang J, Jackson SG, Wardale J, Jones SW. Hypoxia modulates the phenotype of osteoblasts isolated from knee osteoarthritis patients, leading to undermineralized bone nodule formation. Arthritis Rheumatol. 2014;66(7): 1789-99.

26. Burkhardt D, Hwa SY, Ghosh P. A novel microassay for the quantitation of the sulfated glycosaminoglycan content of histological sections: its application to determine the effects of Diacerhein on cartilage in an ovine model of osteoarthritis. Osteoarthr Cartil. 2001;9(3):238-47.

27. Bradford MM. A rapid and sensitive method for the quantitation of microgram quantities of protein utilizing the principle of protein-dye binding. Anal Biochem. 1976;72:248-54.

28. Gregory CA, Gunn WG, Peister A, Prockop DJ. An alizarin red-based assay of mineralization by adherent cells in culture: comparison with cetylpyridinium chloride extraction. Anal Biochem. 2004;329(1):77-84.

29. Deuis JR, Dvorakova LS, Vetter I. Methods used to evaluate pain behaviors in rodents. Front Mol Neurosci. 2017;10:284

30. Maruotti N, Corrado A, Cantatore FP. Osteoblast role in osteoarthritis pathogenesis. J Cell Physiol. 2017;232(11):2957-63.

31. Benya PD, Padilla SR, Nimni ME. Independent regulation of collagen types by chondrocytes during the loss of differentiated function in culture. Cell. 1978;15(4):1313-21.

32. Cooke M, Pearson MJ, Williams RL, Grover LM, Jones SW. Characterisation of the biochemical and biophysical properties of biomimetic cartilage models. Osteoarthr Cartilage. 2016;24:S172-3.

33. von der Mark K, Gauss V, von der Mark H, Muller P. Relationship between cell shape and type of collagen synthesised as chondrocytes lose their cartilage phenotype in culture. Nature. 1977;267(5611):531-2.
34. Kuyinu EL, Narayanan G, Nair LS, Laurencin CT. Animal models of osteoarthritis: classification, update, and measurement of outcomes. J Orthop Surg Res. 2016;11:19.

35. Radin EL. Subchondral bone changes and cartilage damage. Equine Vet J. 1999;31(2):94-5.

36. Radin EL, Abernethy PJ, Townsend PM, Rose RM. The role of bone changes in the degeneration of articular cartilage in osteoarthrosis. Acta Orthop Belg 1978;44(1):55-63.

37. Felson DT, McLaughlin S, Goggins J, LaValley MP, Gale ME, Totterman S, Li W, Hill C, Gale D. Bone marrow edema and its relation to progression of knee osteoarthritis. Ann Intern Med. 2003;139(5 Pt 1):330-6.

38. Hunter DJ, Zhang Y, Niu J, Goggins J, Amin S, LaValley MP, Guermazi A, Genant H, Gale D, Felson DT. Increase in bone marrow lesions associated with cartilage loss: a longitudinal magnetic resonance imaging study of knee osteoarthritis. Arthritis Rheum. 2006;54(5):1529-35.

39. Neogi T. Clinical significance of bone changes in osteoarthritis. Ther Adv Musculoskelet Dis. 2012;4(4):259-67.

40. Felson DT, Chaisson CE, Hill CL, Totterman SM, Gale ME, Skinner KM, Kazis L, Gale DR. The association of bone marrow lesions with pain in knee osteoarthritis. Ann Intern Med. 2001;134(7):541-9.

41. Hunter DJ, Gerstenfeld L, Bishop G, Davis AD, Mason ZD, Einhorn TA, Maciewicz RA, Newham P, Foster M, Jackson S, et al. Bone marrow lesions from osteoarthritis knees are characterized by sclerotic bone that is less well mineralized. Arthritis Res Ther. 2009;11(1):R11.

42. Anderson-Mackenzie JM, Quasnichka HL, Starr RL, Lewis EJ, Billingham ME, Bailey AJ. Fundamental subchondral bone changes in spontaneous knee osteoarthritis. Int J Biochem Cell Biol. 2005;37(1):224-36.

43. Quasnichka HL, Anderson-MacKenzie JM, Bailey AJ. Subchondral bone and ligament changes precede cartilage degradation in Guinea pig osteoarthritis. Biorheology. 2006;43(3,4):389-97.

44. Hilal G, Martel-Pelletier J, Pelletier JP, Ranger P, Lajeunesse D. Osteoblast-like cells from human subchondral osteoarthritic bone demonstrate an altered phenotype in vitro: possible role in subchondral bone sclerosis. Arthritis Rheum. 1998;41(5):891-9.

45. Sanchez C, Deberg MA, Bellahcene A, Castronovo V, Msika P, Delcour JP, Crielaard JM, Henrotin YE. Phenotypic characterization of osteoblasts from the sclerotic zones of osteoarthritic subchondral bone. Arthritis Rheum. 2008:58(2):442-55.

46. Couchourel D, Aubry I, Delalandre A, Lavigne M, Martel-Pelletier J, Pelletier JP, Lajeunesse D. Altered mineralization of human osteoarthritic osteoblasts is attributable to abnormal type I collagen production. Arthritis Rheum. 2009;60(5):1438-50.

47. Gao J, Feng Z, Wang X, Zeng M, Liu J, Han S, Xu J, Chen L, Cao K, Long J, et al. SIRT3/SOD2 maintains osteoblast differentiation and bone formation by regulating mitochondrial stress. Cell Death Differ. 2018; 25(2):229-40.

48. Tejerina S, De Pauw A, Vankoningsloo S, Houbion A, Renard P, De Longueville F, Raes M, Arnould T. Mild mitochondrial uncoupling induces 3T3-L1 adipocyte de-differentiation by a PPARgamma-independent mechanism, whereas TNFalpha-induced de-differentiation is PPARgamma dependent. J Cell Sci. 2009;122(Pt 1):145-55.

49. Blanco FJ, Rego I, Ruiz-Romero C. The role of mitochondria in osteoarthritis, Nat Rev Rheumatol. 2011;7(3):161-9.

50. Johnson K, Jung A, Murphy A, Andreyev A, Dykens J, Terkeltaub R. Mitochondrial oxidative phosphorylation is a downstream regulator of nitric oxide effects on chondrocyte matrix synthesis and mineralization. Arthritis Rheum. 2000;43(7):1560-70.

51. Maneiro E, Martin MA, de Andres MC, Lopez-Armada MJ, Fernandez-Sueiro $J$, del Hoyo P, Galdo F, Arenas J, Blanco FJ. Mitochondrial respiratory activity is altered in osteoarthritic human articular chondrocytes. Arthritis Rheum. 2003:48(3):700-8.

52. Ding G, Zhao J, Jiang D. Allicin inhibits oxidative stress-induced mitochondrial dysfunction and apoptosis by promoting PI3K/AKT and CREB/ERK signaling in osteoblast cells. Exp Ther Med. 2016;11(6): 2553-60.

53. Pascual-Garrido C, McNickle AG, Cole BJ. Surgical treatment options for osteochondritis dissecans of the knee. Sports Health. 2009;1(4):326-34.

54. Jones SW, Brockbank SM, Clements KM, Le Good N, Campbell D, Read SJ, Needham MR, Newham P. Mitogen-activated protein kinase-activated protein kinase 2 (MK2) modulates key biological pathways associated with OA disease pathology. Osteoarthr Cartil. 2009;17(1):124-31. 
55. Jones SW, Brockbank SM, Mobbs ML, Le Good NJ, Soma-Haddrick S, Heuze AJ, Langham CJ, Timms D, Newham P, Needham MR. The orphan G-protein coupled receptor RDC1: evidence for a role in chondrocyte hypertrophy and articular cartilage matrix turnover. Osteoarthr Cartil. 2006;14(6):597-608.

56. Bian L, Lima EG, Angione SL, Ng KW, Williams DY, Xu D, Stoker AM, Cook JL, Ateshian GA, Hung CT. Mechanical and biochemical characterization of cartilage explants in serum-free culture. J Biomech. 2008;41(6):1153-9

\section{Ready to submit your research? Choose BMC and benefit from:}

- fast, convenient online submission

- thorough peer review by experienced researchers in your field

- rapid publication on acceptance

- support for research data, including large and complex data types

- gold Open Access which fosters wider collaboration and increased citations

- maximum visibility for your research: over $100 \mathrm{M}$ website views per year

At $\mathrm{BMC}$, research is always in progress.

Learn more biomedcentral.com/submissions 